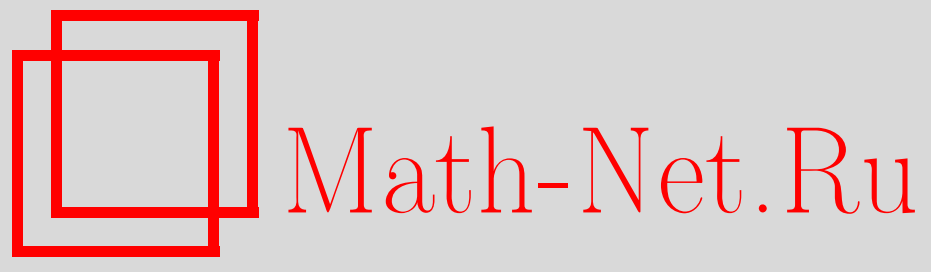

М.-М. Деза, М. И. Штогрин, Новые примеры обобщенных фуллеренов, УМН, 2009, том 64, выпуск 1, 145-146

DOI: https://doi.org/10.4213/rm9268

Использование Общероссийского математического портала Math-Net.Ru подразумевает, что вы прочитали и согласны с пользовательским соглашением http://www . mathnet.ru/rus/agreement

Параметры загрузки:

IP: 54.166 .219 .16

26 апреля 2023 г., 02:11:18

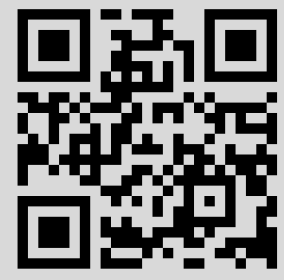




\title{
Новые примеры обобщенных фуллеренов
}

\author{
М. Деза, М. И. Штогрин
}

Фуллерен - простой (3-валентный) полиэдр, грани которого суть пентагоны и гексагоны; а значит, число пентагонов равно 12. Лишь 4 фуллерена не имеют смежных гексагонов - это додекаэдр $F_{20}$, гексагональная бочка $F_{24}$ (чьи два гексагона разделены двумя поясами из пентагонов), фуллерен $F_{26}$ (единственный с 26 вершинами) и фуллерен $F_{28}$ (один из двух с 28 вершинами); они имеют соответственно $0,2,3$ и 4 несмежных гексагонов, 20, 24, 26 и 28 вершин и называются фуллеренами ФранкаКаспера, сокращенно FK-фуллеренами. Удлиненная бочка $F_{36}$ получается из $F_{24}$ добавлением пояса из 6 гексагонов между поясами из пентагонов. Удлиненный додекаэдр $F_{30}$ получается из $F_{20}$ добавлением пояса из 5 гексагонов. Скрученный додекаэдр $F_{20}^{\prime}$ (родственный простой полиэдр с тройной осью, но не фуллерен) имеет пояс из чередующихся трех гексагонов и трех квадратов; остальные 6 граней суть пентагоны.

$\mathbb{R}^{3}$-фуллереном (space fullerene) называется простое (т. е. 4-валентное) нормальное разбиение пространства $\mathbb{R}^{3}$ на фуллерены. $\mathbb{R}^{3}$-фуллерен называется кристаллическим, если его группа симметрии является кристаллографической - обладает трансляциями в трех некомпланарных направлениях. FK- $\mathbb{R}^{3}$-фуллерены (или фазы Франка-Kaспера) - это кристаллические $\mathbb{R}^{3}$-фуллерены, чьи ячейки суть FK-фуллерены (все 4 или их часть), см. [1]-[3]. Ровно 24 FK-фазы известны как металлические сплавы. В [2], [3] было замечено, что пропорции ячеек $F_{20}, F_{24}, F_{26}, F_{28}$ в каждом из 24 случаев являются линейными комбинациями пропорций для основных примеров $A_{15}, C_{15}$ и $Z$ с пропорциями $2: 6: 0: 0,16: 0: 0: 8,3: 2: 2: 0$. Однако в 2008 г. M. Дютур, М. Деза и О. Дельгадо-Фридрихс нашли все FK- $\mathbb{R}^{3}$-фуллерены с не более чем 16 ячейками в фундаментальной области, из них 3 с пропорциями $3: 4: 2: 0$, $7: 4: 2: 2,6: 6: 4: 0$ суть контрпримеры к нерешенной в то время общей гипотезе.

Единственный известный кристаллический $\mathbb{R}^{3}$-, но не $\mathrm{FK}-\mathbb{R}^{3}$-фуллерен построен в [4]; он был рассмотрен затем в [5] в деталях как разбиение Деза-Штогрина. Назовем его DS- $\mathbb{R}^{3}$-фуллереном; он состоит из $F_{20}, F_{24}, F_{36}$ в пропорции $7: 2: 1$ и имеет группу симметрии $P 6 / \mathrm{mmm}$. Все кристаллические $\mathbb{R}^{3}$-фуллерены с не более чем 7 орбитами вершин суть $A_{15}, C_{15}, Z, C_{14}$ и DS- $\mathbb{R}^{3}$-фуллерен, имеющие соответственно $3,3,5,7$ и 7 орбит, см. [5]. DS- $\mathbb{R}^{3}$-фуллерен оказался лучшим среди известных в смысле среднего числа сторон его граней, 56/11, ближайшего к недостижимому минимуму 5.

$\mathrm{C} \mathbb{R}^{3}$-фуллеренами тесно связано разбиение с группой $P 6 / \mathrm{mmm}$, соответствующее клатрату $H$. Его фундаментальная область имеет 34 вершины (молекулы воды), образующие ячейки $F_{20}, F_{20}^{\prime}, F_{36}$ в пропорции $3: 2: 1$, см. [6]. Клатраты - это кристаллические твердые субстанции, основанные на кристаллической воде (они похожи на лед), в которой неполярные молекулы (обычно газы) пойманы внутри ячеек молекул воды с водородными связями. Базовые клатраты типа I ( $A_{15}$ в кристаллографической терминологии; они имеют группу $P m \overline{3} n)$ и типа II $\left(C_{15}\right.$ в кристаллографической терминологии; они имеет группу $F d \overline{3} m)$ соответствуют $\mathrm{FK}-\mathbb{R}^{3}$-фуллеренам $A_{15}$ и $C_{15}$. Кристаллический $\mathrm{FK}-\mathbb{R}^{3}$-фуллерен $C_{14}$ имеет группу $P 6_{3} / m m c$, чья фундаментальная область состоит из восьми $F_{20}$ и четырех $F_{28}$. По существу $C_{14}$ - это гексагональная версия $C_{15}$, с той же самой пропорцией $2: 0: 0: 1$, но с 12 ячейками в фундаментальной области группы $P 6_{3} / m m c$. Фуллерены $F_{28}$ образуют структуру алмаза (diamond) в $C_{15}$ и структуру гексагонального алмаза (lonsdaleite) в $C_{14}$.

Ниже из любого кристаллического $\mathbb{R}^{3}$-фуллерена с осью 6-го порядка и плоскостями зеркальной симметрии вдоль нее построено бесконечное множество некристалли-

Работа выполнена при поддержке РФФИ (проект 08-01-90102-Мол), программы “Ведущие научные школы" (проект НШ-4182.2006.1) и государственной программы фундаментальных исследований ОМН РАН “Современные проблемы теоретической математики”. 
ческих $\mathbb{R}^{3}$-фуллеренов. Этот способ построения $\mathbb{R}^{3}$-фуллеренов является трехмерным аналогом представленного ниже метода получения $\mathbb{R}^{2}$-фуллеренов.

Обобщенный фуллерен (конкретнее, $\mathbb{M}^{n}$-фуллерен, см. [4]) - это простое разбиение многообразия $\mathbb{M}^{n}$ любой размерности $n$ (замкнутого или неограниченного), чьи двумерные грани суть пентагоны и гексагоны. Обычный фуллерен есть $\mathbb{S}^{2}$-фуллерен. Обобщенный $\mathbb{P}^{2}$-фуллерен, где $\mathbb{P}^{2}$ - проективная плоскость, имеет 6 пентагонов.

Теперь дадим способ построения $\mathbb{R}^{2}$-фуллеренов, гомеоморфных плоскости, которые имеют не более 6 пентагонов. Возьмем стандартное разбиение плоскости $\mathbb{R}^{2}$ на гексагоны. Центр одного из гексагонов примем за вершину угла $\pi / 3$, чьи граничные лучи направим по осям зеркальной симметрии. Сделаем следующую операцию: удалим внутренность угла $\pi / 3$, а граничные лучи отождествим поворотом на угол $\pi / 3$. После этой операции одни половины гексагонов удалятся вместе с внутренностью угла, а другие половины гексагонов склеятся в целый гексагон. Лишь один гексагон, в центре которого выбрана вершина угла $\pi / 3$, превратится после удаления его шестой части в пентагон. В итоге получится простое нормальное разбиение плоскости на гексагоны и один пентагон. Этот простейший $\mathbb{R}^{2}$-фуллерен имеет группу $5 \mathrm{~m}$. Точно так же в этом $\mathbb{R}^{2}$-фуллерене любой гексагон можно опять заменить на пентагон. Для этого нужно удалить тот угол $\pi / 3$ с вершиной в центре гексагона, внутри которого не лежит уже имеющийся пентагон. Так получится бесконечное множество $\mathbb{R}^{2}$-фуллеренов с двумя пентагонами; группой каждого из этих $\mathbb{R}^{2}$-фулеренов является $2 m$ или 2 . Операцию можно повторить шесть раз, так как из разбиения евклидовой плоскости на гексагоны можно удалить шесть попарно непересекающихся плоских углов $\pi / 3$, но не более, что следует из равенства $6 \cdot \pi / 3=2 \pi$.

$\mathrm{DS}-\mathbb{R}^{3}$-фуллерен имеет группу $P 6 / \mathrm{mmm}$. Построим двугранный угол $\pi / 3$, составленный плоскостями зеркальной симметрии. Сделаем следующую операцию: удаляем внутренность угла, а грани отождествляем (поворотом на $\pi / 3$ ). После этой операции одни половины фуллеренов удалятся, а другие половины склеятся в целые фуллерены. Лишь те фуллерены, которые пронизывает ребро двугранного угла $\pi / 3$, после удаления шестой части из каждого превратятся в другие фуллерены: $F_{36}$ в $F_{30}$, $F_{24}$ в $F_{20}$. Получится простое нормальное разбиение пространства $\mathbb{R}^{3}$ на фуллерены $F_{36}, F_{30}, F_{24}$ и $F_{20}$. Это новый $\mathbb{R}^{3}$-фуллерен. Его группа симметрии уже не кристаллографическая, а стержневая, $p 5 / \mathrm{mmm}$, с одним трансляционным периодом. В новом $\mathbb{R}^{3}$-фуллерене вдоль произвольной локальной оси 6-го порядка можно опять заменить $F_{36}$ на $F_{30}$, а $F_{24}$ на $F_{20}$. Для этого нужно удалить тот двугранный угол $\pi / 3$ с ребром вдоль локальной оси 6-го порядка, внутри которого не лежит возникший фуллерен $F_{30}$. Так получим бесконечное множество $\mathbb{R}^{3}$-фуллеренов с двумя локальными стержневыми группами с осью 5 -го порядка; каждый имеет стержневую группу $p m m m$ или $p 2 / m 11$. Операцию можно повторить 6 раз. Это и есть трехмерный аналог.

Теорема. Так строятся $\mathbb{R}^{3}$-фуллерены из всех других с группой $\mathrm{P} 6 / \mathrm{mmm}$.

\section{Список литературы}

[1] F. C. Frank, J.S. Kasper, Acta Cryst., 11:3 (1958), 184-190; 12:7 (1959), 483-499. [2] Я. П. Ярмолюк, П. И. Крипякевич, Кристаллография, 19 (1974), 539-545. [3] D. P. Shoemaker, B. Shoemaker, Acta Cryst., B42 (1986), 3-11. [4] M. Deza, M. I. Shtogrin, Southeast Asian Bull. Math., 23:1 (1999), 9-18. [5] O. Delgado-Friedrichs, M. O'Keeffe, Acta Cryst., A62:3 (2006), 228-229. [6] E. D. Sloan, Jr., Clathrate hydrates of natural gases, Dekker, New York, 1998.

\section{М. Деза (M. Deza)}

École Normale Supérieure, Paris; JAIST, Japan

E-mail: Michel.Deza@ens.fr

М. И. Штогрин (M. I. Shtogrin)

Математический институт им. В. А. Стеклова РАН

E-mail: stogrin@mi.ras.ru
Представлено В. М. Бухштабером Принято редколлегией 07.12 .2008 